\title{
Publisher's Note: Asymptotic gravitational wave fluxes \\ from a spinning particle in circular equatorial orbits around a rotating black hole \\ [Phys. Rev. D 93, 044015 (2016)]
}

Enno Harms, Georgios Lukes-Gerakopoulos, Sebastiano Bernuzzi, and Alessandro Nagar

Q (Received 22 October 2019; published 2 December 2019)

DOI: $10.1103 /$ PhysRevD.100.129901

This paper was published online on 5 February 2016 with an error in Eq. (19). The equation has been corrected as of 21 November 2019. The equation is incorrect in the printed version of the journal. 